\title{
JUGER DE L'INCONSTITUTIONNALITÉ D'UNE NORME CONSTITUTIONNELLE ? LE CAS DE LA DÉCISION DU TRIBUNAL CONSTITUTIONNEL PLURINATIONAL DE BOLIVIE DU 28 NOVEMBRE 2017
}

\section{Victor Audubert}

Presses Universitaires de France | « Revue française de droit constitutionnel » 2019/4 N 120 | pages e1 à e17

ISSN 1151-2385

ISBN 9782130821830

\section{Article disponible en ligne à l'adresse :}

https://www.cairn.info/revue-francaise-de-droit-constitutionnel-2019-4-page-e1.htm

Distribution électronique Cairn.info pour Presses Universitaires de France.

(C) Presses Universitaires de France. Tous droits réservés pour tous pays.

La reproduction ou représentation de cet article, notamment par photocopie, n'est autorisée que dans les limites des conditions générales d'utilisation du site ou, le cas échéant, des conditions générales de la licence souscrite par votre établissement. Toute autre reproduction ou représentation, en tout ou partie, sous quelque forme et de quelque manière que ce soit, est interdite sauf accord préalable et écrit de l'éditeur, en dehors des cas prévus par la législation en vigueur en France. Il est précisé que son stockage dans une base de données est également interdit. 


\section{Juger de l'inconstitutionnalité d'une norme constitutionnelle? Le cas de la décision du Tribunal constitutionnel plurinational de Bolivie du 28 novembre 2017}

VICTOR AUDUBERT

La sentence constitutionnelle plurinationale $\mathrm{n}^{\circ}$ 0084/2017 du Tribunal constitutionnel plurinational (TCP) du 28 novembre 2017, dans une décision longue de 81 pages, décide de l'inconstitutionnalité de plusieurs articles de la Loi sur le régime électoral ${ }^{1}$ et supprime plusieurs articles de la Constitution politique de l'État (CPE) de 2009, en particulier l'article 168 qui limite le nombre de mandats présidentiels : « La période du mandat de la Présidente ou du Président et de la Vice-Présidente ou du Vice-Président de l'État est de cinq ans, et ils peuvent être réélus une seule fois de manière continue ${ }^{2}$. » Pour ce faire, le TCP s'appuie sur la supposée incompatibilité de la Constitution bolivienne avec la Convention interaméricaine des droits de l'Homme (CIDH). En effet, la Constitution bolivienne dispose, dans son article 256, que les traités internationaux, s'ils contiennent des dispositions relatives aux droits de l'Homme plus favorables que la Constitution, prévalent sur cette dernière.

Or, le TCP estime que l'article 23 de la CIDH, qui dispose notamment que n'importe quel citoyen peut accéder et participer aux fonctions électives, prévaut sur les dispositions contenues dans la Constitution bolivienne ${ }^{3}$. Du fait d'une interprétation très extensive de cet article, le TCP en conclut que la limitation du mandat présidentiel - et par extension n'importe quel mandat exécutif - est contraire aux traités internationaux.

Victor Audubert, docteur en droit public, CERAL, université Paris XIII, Sorbonne Paris Cité.

1. Articles 52.II, 64, 65, 71 et 72 de la Loi n 26 sur le régime électoral du 30 juin 2010.

2. Article 168 de la CPE de 2009.

3. "Tous les citoyens doivent jouir des droits et facultés ci-après énumérés : De participer à la direction des affaires publiques, directement ou par l'intermédiaire de représentants librement élus ; d'élire et d'être élus dans le cadre de consultations périodiques authentiques, tenues au suffrage universel et égal, et par scrutin secret garantissant la libre expression de la volonté des électeurs, et d'accéder, à égalité de conditions générales, aux fonctions publiques de leur pays ", article 23 de la Convention interaméricaine des droits de l'homme. Disponible sur : https://www.cidh.oas.org/basicos/french/c.convention.htm 
On constate que le TCP, en tant que juge constitutionnel, s'octroie le pouvoir de modifier la Constitution. Or, il n'est qu'un organe constitué, ne disposant pas d'un pouvoir constituant ; celui-ci appartient normalement au peuple. Seul ce dernier peut, par la voie du référendum, décider de modifier la Constitution, voire d'en changer ${ }^{4}$. Par ailleurs, on remarque que le juge constitutionnel bolivien interprète de manière controversée une disposition de la $\mathrm{CIDH}$, et place celle-ci au-dessus de la Constitution bolivienne.

Cette décision nous apparaît fondamentale, en ce que le juge constitutionnel bolivien s'octroie des pouvoirs extra-constitutionnels - c'est-àdire qui ne sont pas prévus par la Constitution, voire qui s'y opposent - en se fondant sur des méthodes interprétatives très audacieuses.

Il s'agira, dans cet article, d'étudier les arguments mobilisés par le TCP dans sa décision contre la limitation des mandats (I), avant de nous pencher sur les méthodes interprétatives développées par le juge constitutionnel qui aboutissent à la suppression de plusieurs articles du texte constitutionnel (II).

\section{I - UNE DÉCISION CONTRE LA LIMITATION DU MANDAT DES ORGANES EXÉCUTIFS}

La Constitution politique de l'État (CPE) en 2009, limite à deux le nombre de mandats présidentiels consécutifs dans le temps, contrairement au précédent texte constitutionnel qui interdisait la réélection immédiate du Président de la République.

Cette limitation du cumul des mandats présidentiels dans le temps est le fruit d'un accord politique entre le MAS (Movimiento Al Socialismo, le parti politique soutenant Evo Morales) et l'opposition durant le processus constituant bolivien ${ }^{5}$. Cet accord politique comporte une modification discrète, avec l'ajout d'une Disposition transitoire première dans le texte constitutionnel. Celle-ci dispose, dans son second alinéa, que les mandats antérieurs à la présente Constitution seront pris en compte dans

4. Selon l'article 411 de la CPE de 2009, le texte constitutionnel peut être révisé de deux manières. S'il s'agit d'une révision partielle, l'initiative peut être populaire avec la signature d'au moins $20 \%$ du corps électoral, ou de l'Assemblée législative plurinationale et doit être approuvée aux deux-tiers des membres présents. S'il s'agit d'une révision totale, « qui touche aux bases fondamentales, aux droits, devoirs et garanties, ou à la suprématie et la révision de la Constitution ", il est nécessaire de convoquer une Assemblée constituante. La convocation peut être à l'initiative de l'ALP, de $20 \%$ du corps électoral, et du Président de l'État. Dans les deux cas, le nouveau texte constitutionnel est soumis au référendum.

5. Le processus constituant bolivien a débuté le 6 août 2006, et s'est achevé avec la promulgation de la nouvelle Constitution politique de l'État le 7 février 2009. 
le décompte des nouveaux mandats - ce qui implique alors le renoncement du Président et du Vice-président actuels à une réélection en $2014^{6}$. Nous allons voir que la protection des droits politiques est mobilisée par le juge constitutionnel (A) en prenant appui sur la Convention interaméricaine des droits de l'Homme (B).

\section{A - LA PROTECTION DES DROITS POLITIQUES CONTRE LA LIMITATION DU MANDAT PRÉSIDENTIEL}

L'action d'inconstitutionnalité est portée par des députés et sénateurs du MAS. Elle a été présentée le 18 septembre 2017 au TCP, qui l'a accepté le 28 septembre avec la décision AC 0269/2017-CA. Cette action demande la reconnaissance de l'inconstitutionnalité des articles 52.II, 64, 65, 71 et 72 de la LRE ainsi que la non-application des articles 156, 168, 285.II et 288 de la CPE au regard des articles 26 et 28 du même texte et des articles 1.1, 23, 24 et 29 de la CIDH. Cette action s'appuie en particulier sur les articles 410.II et 256.I de la CPE, qui intègrent les conventions internationales relatives aux droits humains dans le bloc de constitutionnalité bolivien, et qui reconnaissent que les dispositions conventionnelles plus favorables que les dispositions constitutionnelles s'appliquent au détriment de ces dernières. Il existe donc, selon les députés, un "paradoxe » :

« Il existe un paradoxe à l'intérieur du texte constitutionnel lui-même, car d'une part il reconnaît les droits politiques de citoyens et leur possibilité d'être candidats et de se présenter à des élections propres et justes dans les articles 26 et 28 de la CPE et d'autre part, les articles 156, 168, 285.II et 288 de la même norme suprême limitent lesdits droits, s'éloignant de ce fait du texte constitutionnel et des traités et conventions internationales ratifiées par l'État bolivien, comme le Pacte de San José ${ }^{7}$ connu sous le nom de Convention interaméricaine des droits de l'Homme $[\ldots]^{8}$. »

Ces droits politiques sont définis de la manière suivante par les députés du MAS :

6. « Los mandatos anteriores a la vigencia de esta Constitución serán tomados en cuenta a los efectos del cómputo de los nuevos periodos de funciones », Disposition transitoire première de la CPE de 2009.

7. Cette convention a été ratifiée par la Bolivie avec la loi no 1340 du 11 février 1993.

8. "Asimismo, se evidencia una "paradoja" dentro del propio texto constitucional, pues por una parte reconoce los derechos políticos de las y los ciudadanos y su posibilidad de ser candidatos y postularse a elecciones limpias y justas en los arts. 26 y 28 de la CPE y por otra parte, los arts. 156, 168, 285.II y 288 de la misma Norma Suprema, limitan dichos derechos, alejándose de esta manera del propio texto constitucional y de los Tratados y Convenios internacionales suscritos por el Estado boliviano, como el "Pacto de San José de Costa Rica" hoy Convención Americana sobre Derechos Humanos [...] », sentence constitutionnelle plurinationale $\mathrm{n}^{\circ}$ 0084/2017, Tribunal constitutionnel plurinational, Sucre, 28 novembre 2017. 
«Les droits politiques sont en même temps le revers essentiel d'autres droits fondamentaux des personnes, comme l'interdiction de toute forme de discrimination, lesquels doivent être exercés sans aucune restriction indue et qui comprennent de manière indivisible les droits essentiels de participer aux sujets politiques directement ou par la voie de ses représentants, de voter et d'être élu dans les élections prévues, authentiques, réalisées par le suffrage universel et égal et par le suffrage secret qui garantissent la libre expression de la volonté des électeurs ${ }^{9}$. »

Le TCP reprend point par point l'argumentation développée par les députés du MAS dans leur action en inconstitutionnalité, en particulier dans le titre III sur les fondements juridiques de la décision. Le juge constitutionnel bolivien, dans la sixième partie de son titre III, revient sur l'interprétation de la Constitution par le TCP. Il s'appuie notamment sur l'article 196.II de la CPE qui dispose que les critères d'interprétation se trouvent dans la volonté du constituant (interprétation exégétique) ainsi que la teneur littérale du texte (interprétation grammaticale). Bien qu'il reconnaisse d'autres méthodes interprétatives ${ }^{10}$, il fonde sa décision sur l'interprétation exégétique, à partir de la volonté du constituant, en particulier dans les travaux de l'Assemblée constituante. Les sources mobilisées sont principalement l'encyclopédie historique documentaire publiée en $2009^{11}$ qui réunit l'ensemble des travaux, débats et motions des commissions. Le juge constitutionnel s'appuie sur ce document et en particulier sur des propositions de la commission en charge de l'organe exécutif :

« Le présent article introduit la réélection du Président et du Vice-Président. Cela se fonde sur le fait que n'importe quel(le) Président(e) qui a obtenu de bons résultats dans son action gouvernementale et qui a bénéficié d'un soutien majoritaire de la population; le souverain décidera seul. La réélection immédiate par la volonté du peuple est une nouvelle possibilité de rénover la confiance déposée dans un représentant au travers du vote citoyen, en accord avec le respect de l'offre électoral. De cette manière la gouvernabilité est durable

9. «Los derechos políticos al mismo tiempo son reverso esencial de otro derecho fundamental de las personas, como ser la prohibición de toda forma de discriminación, por lo que aquéllos deben ser ejercidos sin ninguna restricción indebida y que comprenden indivisiblemente derechos esenciales tales como a participar en la dirección de los asuntos públicos directamente o por medio de sus representantes, a votar y ser elegido en elecciones periódicas, auténticas, realizadas por sufragio universal e igual y por voto secreto que garantice la libre expresión de la voluntad de los electores »; sentence constitutionnelle plurinationale $\mathrm{n}^{\circ}$ 0084/2017, Tribunal constitutionnel plurinational, Sucre, 28 novembre 2017.

10. Sentence constitutionnelle plurinationale $n^{\circ} 0850 / 2013$, Tribunal constitutionnel plurinational, Sucre, 17 juin 2013.

11. Juan Carlos Pinto Quintanilla (coord.), Enciclopedia histórica documental del Proceso constituyente boliviano, La Paz, Vice-Présidence de l'État plurinational de Bolivie, 2009. 
étant donné que la continuité du travail déjà réalisé pour la réalisation des meilleures opportunités et le développement permanent du pays ${ }^{12}$. »

Cependant, il ne s'agit pas là d'une version intermédiaire ou même définitive du projet de loi constitutionnelle, mais d'une simple proposition de la commission contenue dans le Rapport majoritaire de la commission. De ce fait, le juge constitutionnel s'appuie non pas sur les projets constitutionnels votés à Sucre ou à Oruro, mais sur des rapports des différentes commissions de l'Assemblée constituante. Il considère ainsi que la volonté du constituant se trouve dans ces débats. Or, la volonté du constituant, si elle a un temps été exprimée par l'Assemblée constituante, l'est en définitive par le peuple via le référendum de 2009.

La décision du 28 novembre 2017 revient donc sur le processus constituant bolivien en affirmant que les constituants, dans le rapport majoritaire de la commission $\mathrm{n}^{\mathrm{o}} 7$ en charge de l'organe exécutif, n'avaient pas introduit de limitation de mandat. Ils citent donc la commission $\mathrm{n}^{\circ} 7$ qui aurait proposé la formulation suivante au sujet du mandat présidentiel : "La Présidente ou le Président et la Vice-Présidente ou le Vice-Président pourront être réélus consécutivement par la volonté du peuple ${ }^{13}$. » Le juge constitutionnel cite également les commissions $\mathrm{n}^{\circ} 2$ et $\mathrm{n}^{\mathrm{o}} 3$ de l'Assemblée constituante qui n'auraient pas établi de limites relatives à l'élection des autres autorités politiques.

Or, il se trouve que si la commission $\mathrm{n}^{\circ} 7 \mathrm{a} \mathrm{pu}$ formuler de telles propositions, les projets de constitution adoptés à Oruro le 9 décembre 2007 ainsi que par le Congrès national en octobre 2008 n'ont à aucun moment supprimé ou assoupli la limitation de mandat présidentiel; au contraire, cette limitation était partagée par la plupart des constitutionnalistes, ainsi que des organisations indigénistes ${ }^{14}$. Le peuple bolivien,

12. «El presente artículo, introduce la figura de la reelección del Presidente y Vicepresidente, esto se funda en el hecho de que cualquier Presidente (a) que haya logrado resultados óptimos en su Plan de Gobierno y beneficie de forma mayoritaria a la población, como justo reconocimiento será el soberano quien decida. La reelección consecutiva por voluntad del pueblo es la nueva posibilidad de renovar confianza depositada en un representante mediante el voto ciudadano, de acuerdo al cumplimiento de su oferta electoral. De esta manera la gobernabilidad es sostenible dando continuidad al trabajo ya realizado para el logro de mayores oportunidades y el desarrollo permanente del país »; Juan Carlos Pinto Quintanilla (coord.), Enciclopedia historica documental del Proceso constituyente boliviano. Tomo III: Informes por comisión, op. cit., p. 910.

13. «La Presidenta o Presidente y Vicepresidenta o Vicepresidente, podrán ser reelectos consecutivamente por voluntad del pueblo »; Juan Carlos Pinto Quintanilla (coord.), Enciclopedia historica documental del Proceso constituyente boliviano. Tomo III: Informes por comisión, op. cit., p. 985.

14. Notamment par le Pacte d'Unité qui a milité pour une seule réélection, comme le souligne l'article 86 de son projet de Constitution : «El mandato del o la Presidente y del o la Vicepresidente es de 5 años y podrán ser reelectos o reelectas por una sola vez para un nuevo período constitucional »; El Pacto de Unidad y el Proceso de Construcción de una Propuesta de Constitución Política del Estado, Pacto de Unidad, La Paz, p. 185. 
lors du référendum constituant de 2009, a pour sa part approuvé une Constitution où figure cette limitation du mandat présidentiel. Le TCP suppose donc que la volonté des constituants est à chercher dans la rédaction d'un rapport majoritaire, c'est-à-dire un rapport approuvé la majorité des membres d'une commission. Cependant, la volonté du peuple bolivien, et donc du pouvoir constituant, s'est exprimée sur ce point en faveur de la limitation des mandats.

\section{B - LE RECOURS À LA CONVENTION INTERAMÉRICAINE DES DROITS HUMAINS}

On retrouve dans la Constitution bolivienne de 2009 des éléments liés à la méthode d'interprétation des normes constitutionnelles, notamment au regard des normes internationales. Dans l'article 13.IV de la Constitution, il est ainsi indiqué que «les droits et devoirs consacrés dans cette Constitution s'interpréteront en conformité avec les traités internationaux relatifs aux droits humains ratifiés par la Bolivie ${ }^{15}$ ». Les normes doivent ainsi être interprétées selon les traités et conventions internationales relatifs aux droits humains. Par ailleurs, selon l'article 256.I, « les traités et instruments internationaux relatifs aux droits humains qui ont été signés, ratifiés ou ceux auxquelles a adhéré l'État, ceux-là qui contiennent des droits humains plus favorables à ceux contenus dans la Constitution s'appliqueront de manière préférentielle sur ces derniers ${ }^{16} »$. Enfin, l'interprétation extensive et favorable des droits fondamentaux est consacrée dans l'article 29 de la $\mathrm{CIDH}^{17}$.

Le juge constitutionnel bolivien a donc recours à la technique pro bomine dans l'interprétation des dispositions constitutionnelles. Cette technique veut que les droits humains contenus dans les conventions

15. «Los derechos y deberes consagrados en esta Constitución se interpretarán de conformidad con los Tratados Internacionales de derechos humanos ratificados por Bolivia ", article 13.IV de la CPE de 2009.

16. «Los tratados e instrumentos internacionales en materia de derechos humanos que hayan sido firmados, ratificados o a los que se hubiera adherido el Estado, que declaren derechos más favorables a los contenidos en la Constitución, se aplicarán de manera preferente sobre ésta », article 256.I de la CPE de 2009.

17. «Aucune disposition de la présente Convention ne peut être interprétée comme : Autorisant un État partie, un groupement ou un individu à supprimer la jouissance et l'exercice des droits et libertés reconnus dans la présente Convention ou à les restreindre plus qu'il n'est prévu dans ladite Convention; restreignant la jouissance et l'exercice de tout droit ou de toute liberté reconnus par la législation d'un État partie ou dans une convention à laquelle cet État est partie ; excluant d'autres droits et garanties inhérents à la personne humaine ou qui dérivent de la forme démocratique représentative de gouvernement ; supprimant ou limitant les effets que peuvent avoir la Déclaration américaine des droits et devoirs de l'homme et tous autres actes internationaux de même nature », article 29 de la Convention interaméricaine relative aux droits de l'homme. Disponible sur : https:// www.cidh.oas.org/Basicos/French/c.convention.htm 
internationales et qui sont plus favorables que ceux contenus dans le texte constitutionnel prédominent sur ces derniers. C'est cette technique qui est mobilisée dans la décision controversée du 28 novembre 2017 du TCP relative à la réélection présidentielle ${ }^{18}$.

Le juge constitutionnel, dans la décision du 28 novembre 2017, estime que les dispositions contenues dans la Convention interaméricaine des droits humains sont, d'une part, plus favorables que celles contenues dans la Constitution de 2009, et que d'autre part elles s'opposent au principe de limitation du mandat électoral :

«Selon sa vocation internationaliste, le texte constitutionnel avec son article 256 cède à cette hiérarchie en faveur des traités internationaux en matière de droits humains, en reconnaissant de plus qu'il n'est pas nécessaire que ces derniers aient été ratifiés par le pays, qu'ils s'appliquent de manière préférentielle sur la propre Constitution bolivienne et que les droits fondamentaux seront interprétés en accord avec les traités internationaux relatifs aux droits humains ${ }^{19}$. »

La jurisprudence internationale est ainsi incorporée dans le bloc de constitutionnalité bolivien. Le juge constitutionnel renvoie à une précédente décision afin de rappeler l'obligation d'exercer un contrôle de conventionnalité de la part de l'organe judiciaire sur les normes constitutionnelles :

«Les juges et tribunaux ont le devoir d'appliquer la norme qui est la plus favorable pour la protection du droit en question - qu'elle soit contenue dans la Constitution ou dans le bloc de constitutionnalité - et d'adopter l'interprétation qui soit la plus favorable et extensive [...] Ils ont le devoir - en exerçant le contrôle de conventionnalité - de toujours et à n'importe quel moment interpréter le droit en accord aux normes contenues dans les traités et instruments internationaux relatifs aux droits humains ratifiés ou à ceux auxquels l'État a adhéré lorsqu'ils contiennent des droits plus favorables à ceux contenus dans la Constitution politique de l'État [.... À partir de ces critères, ils doivent établir les buts des droits invoqués à partir d'une perspective constitutionnelle et les standards établis par la Cour interaméricaine des droits humains, pour ensuite déterminer si la norme contestée respecte ces derniers ou si elle doit être expulsée de l'ordonnancement juridique ${ }^{20}$. »

18. Voir supra.

19. «Bajo su vocación internacionalista, el propio Texto Constitucional en su art. 256 cede esa jerarquía en favor de los tratados internacionales en materia de derechos humanos, reconociendo además que no es necesario que éstos hayan sido ratificados por el país, que son de preferente aplicación sobre la propia Constitución boliviana y que los derechos fundamentales serán interpretados de acuerdo a los tratados internacionales en materia de derechos humanos »; sentence constitutionnelle plurinationale ${ }^{\circ} 0084 / 2017$, Tribunal constitutionnel plurinational, Sucre, 28 novembre 2017.

20. « Los jueces y tribunales tiene el deber de aplicar aquella norma que sea más favorable para la protección del derecho en cuestión - ya sea que esté contenida en la Constitución o en las normas del bloque de constitucionalidad- y de adoptar la interpretación que sea más 
Une longue partie de ce titre revient sur la nécessité d'effectuer un contrôle de conventionalité au même titre que le contrôle de constitutionnalité : «Les magistrats du Tribunal constitutionnel plurinational se trouvent obligés de réaliser un contrôle de conventionnalité dans les litiges qui lui sont soumis ${ }^{21}$. » Le juge constitutionnel souligne toutefois que cette obligation ne se trouve pas établie de "manière expresse » dans l'ordonnancement juridique national ; ce dernier devient par conséquent le résultat d'engagements internationaux en ayant souscrit et ratifié à la Convention interaméricaine des droits humains par le principe pacta sunt servanda. Il paraît ainsi nécessaire au juge constitutionnel bolivien de « conventionnaliser les normes dans le but d'atteindre une protection effective des droits ${ }^{22} »$. De ce fait, si l'une des normes internes apparaît contraire aux normes conventionnelles, il en résulte « l'inapplication des normes internes contraires à la convention et la jurisprudence de la Cour interaméricaine des droits de l'homme ${ }^{23} »$. On en vient ainsi, selon les mots du juge constitutionnel, à une "Constitution conventionnalisée ».

Le TCP, suivant ce raisonnement pro bomine, en vient donc à rejeter les articles 156, 168, 285.II et 288 de la Constitution bolivienne de 2009 ; il supprime de facto la limitation dans le temps des mandats des organes exécutifs, et en particulier ceux du Président et du Vice-Président de l'État. Cependant, cette interprétation très extensive du contrôle de conventionnalité ainsi que de la lecture de l'article 23 de la $\mathrm{CIDH}$ est loin de faire l'unanimité parmi la doctrine bolivienne, et encore parmi l'OEA. Cette dernière a d'ailleurs estimé que la lecture faite par le TCP de l'article 23 était pour le moins ambiguë ${ }^{24}$.

favorable y extensiva [...] tienen el deber de -ejerciendo el control de convencionalidadinterpretar el derecho de acuerdo a las normas contenidas en tratados e instrumentos internacionales en materia de derechos humanos ratificados o a los que se hubiere adherido el Estado, siempre y cuando, claro está, declaren derechos más favorables a los contenidos en la Constitución Política del Estado [...] Bajo los criterios anotados, se deben establecer los alcances de los derechos alegados desde la perspectiva constitucional y los estándares establecidos por la Corte Interamericana de Derechos Humanos, para luego determinar si la norma impugnada cumple con los mismos o más bien debe ser expulsada del ordenamiento jurídico por ser contraria a ellos » ; sentence constitutionnelle plurinationale $n^{\circ} 0084 / 2017$, Tribunal constitutionnel plurinational, Sucre, 28 novembre 2017.

21. « Los magistrados del Tribunal Constitucional Plurinacional, se encuentran obligados a realizar el control de convencionalidad en el conocimiento de las causas sometidas a su conocimiento »; sentence constitutionnelle plurinationale $n^{\circ} 0084 / 2017$, Tribunal constitutionnel plurinational, Sucre, 28 novembre 2017.

22. «Se debe convencionalizar las mismas a fin de alcanzar una protección efectiva de los derechos »; sentence constitutionnelle plurinationale $\mathrm{n}^{\circ} 0084 / 2017$, Tribunal constitutionnel plurinational, Sucre, 28 novembre 2017.

23. « La inaplicación de la normativa interna contraria a la convención y la jurisprudencia de la Corte Interamericana de Derechos Humanos »; sentence constitutionnelle plurinationale $\mathrm{n}^{\circ} 0084 / 2017$, Tribunal constitutionnel plurinational, Sucre, 28 novembre 2017.

24. José Luis Columba, «Secretario General de la OEA cuestiona fallo del TCP [en ligne] », La Razón, 28 novembre 2017. Disponible sur : http://www.la-razon.com/nacional/ Secretario-General-OEA-cuestiona-TCP_0_2828117219.html 


\section{II - DES MÉTHODES INTERPRÉTATIVES SUI GENERIS DE LA PART DU TCP}

La Constitution bolivienne de 2009 intègre dans son corpus les règles d'interprétation du juge constitutionnel bolivien. Ceci est une originalité de la part du constituant bolivien, car en règle générale ces méthodes d'interprétation sont progressivement dégagées par la jurisprudence constitutionnelle et la doctrine, et non par le constituant lui-même. L'article 196.II dispose ainsi que «dans sa fonction interprétative, le TCP appliquera comme critère d'interprétation, de manière préférentielle, la volonté du constituant en accord avec ses documents, actes et résolutions, ainsi que la teneur littérale du texte ${ }^{25}$ ».

Plusieurs méthodes d'interprétations existent. Certaines prennent en compte la nécessaire évolution du texte constitutionnel, qui doit évoluer en fonction des impératifs de son temps. On retrouve cette interprétation évolutionniste dans la jurisprudence de la Cour suprême des États-Unis. Celle-ci se partage entre les «originalistes» qui prônent de suivre l'intention des Pères fondateurs et de s'en tenir à une lecture littérale ${ }^{26}$, et les «non-originalistes" qui sont les tenants d'une approche dynamique de la Constitution. En ce sens, leur interprétation est sujette à s'adapter aux évolutions de la société ${ }^{27}$.

Dans le droit continental européen, les méthodes d'interprétation renvoient à une herméneutique classique, inspirée des méthodes de Savigny qui prône une interprétation fondée sur la grammaire, la logique et l'histoire. C'est notamment ces méthodes d'interprétation qui ont été reconnues dans la Constitution bolivienne de 2009. Néanmoins, à la suite de Peter Häberle ${ }^{28}$, nous considérons que le seul uage de ces méthodes d'interprétation considère le texte constitutionnel comme une «société fermée". Il faudrait au contraire en faire une «société ouverte », composée de tous ceux qui vivent la norme et l'interprètent, le dernier mot appartenant au juge constitutionnel. La Constitution devient un texte ouvert, toujours de force normative, structurée par la société ouverte.

25. «En su función interpretativa, el Tribunal Constitucional Plurinacional aplicará como criterio de interpretación, con preferencia, la voluntad del constituyente, de acuerdo con sus documentos, actas y resoluciones, así como el tenor literal del texto », article 196.II de la CPE de 2009.

26. Mathieu Carpentier, «Variations autour de l'originalisme », Revue française de droit constitutionnel, 2016/3, n ${ }^{\circ} 107$, p. 739-744.

27. On peut ainsi se référer à la célèbre décision sur l'avortement : décision Roe v. Wade, 410 U.S. 113 (1973). Disponible sur : https://supreme.justia.com/cases/federal/us/410/113/

28. Voir à ce sujet : «Entretien avec le Professeur Peter Häberle [en ligne] », Le nouvel endroit $\mathrm{n}^{\circ}$ 1, 2015, p. 76-81. Disponible sur : https://drive.google.com/file/d/0B_IfWQgd XsG3ZDY1MXJKeDExRjA/view 
À ce sujet, on remarque que le contrôle pluriel de constitutionnalité est reconnu par le TCP lui-même : "L'État plurinational de Bolivie a adopté avec la Constitution politique de l'État de l'année 2009 un système juridictionnel pluriel et concentré de contrôle de constitutionnalité ${ }^{29}$. » Le juge constitutionnel, dans sa décision du 28 novembre 2017, établit une méthode d'interprétation sui generis entendue comme un " processus dynamique, flexible, conciliateur et garantissant les droits fondamentaux et qui s'appliquent au cas concret à la lumière de ses bases principielles, du bloc de constitutionnalité et du contrôle de conventionalité $^{30} »$.

Après avoir étudié la distinction opérée par le juge constitutionnel entre les normes «constitutionnelles-principielles» et les normes « constitutionnelles-règles » $(\mathrm{A})$, il s'agira de constater que le TCP s'est octroyé un pouvoir extraconstitutionnel : celui de supprimer des normes constitutionnelles (B).

\section{A - UN TEXTE CONSTITUTIONNEL DIVISÉ ENTRE NORMES CONSTITUTIONNELLES-PRINCIPIELLES ET CONSTITUTIONNELLES-RÈGLES}

Boris Arias López soutient que le droit bolivien, contrairement aux autres droits, assume sa filiation avec la morale et les valeurs plurielles de la société. De ce fait, si le droit se fonde sur une morale ou une valeur, et que celles-ci fluctuent selon les groupes et le temps, alors le juge doit interpréter le droit différemment selon le contexte culturel et historique, de manière interculturelle - en recherchant les éléments communs - et non multiculturelle - en séparant les éléments différents. Ce processus d'interprétation est fondé sur un dialogue interculturel permanent :

«Il doit être accessible à tous [...] et tous peuvent interpréter les valeurs constitutionnelles $[\ldots]$ afin que les minorités culturelles aient accès et puissent participer à la détermination de la langue, des coutumes, ainsi qu'aux processus de prise de décisions à ceux qui s'en trouvent affectés, ce qui implique que le Tribunal constitutionnel plurinational ne devrait pas pouvoir rendre une décision qui concerne les peuples indigènes sans une consultation préalable ${ }^{31}$.»

29. Déclaration constitutionnelle plurinationale $\mathrm{n}^{\circ}$ 1227/2012, Tribunal constitutionnel plurinational, Sucre, 7 septembre 2012.

30. « [...] como un proceso dinámico, flexible, conciliador y garantistas de los derechos fundamentales y que se aplica al caso concreto a la luz de sus bases principistas, del bloque de constitucionalidad y del control de convencionalidad »; sentence constitutionnelle plurinationale $\mathrm{n}^{\circ}$ 0084/2017, Tribunal constitutionnel plurinational, Sucre, 28 novembre 2017.

31. «El proceso de interpretación debe ser accesible a todos - audiencias temáticas, amicus cuarie, etc. - y todos pueden interpretar los valores constitucionales - sociedad abierta de intérpretes constitucionales de Peter Häberle -, de forma que minorías culturales tengan acceso y participación real en estándares de idioma, costumbres, etc. a los procesos de toma de decisiones que les afecten, lo que implica entonces que el Tribunal Constitucional 
Cependant, après avoir constaté que le juge constitutionnel bolivien souhaite dépasser les méthodes d'interprétation classiques, on peut s'étonner que les contraintes interprétatives, exégétique et grammaticale, soient constitutionnalisées dans le texte constitutionnel de 2009. En effet, ces deux méthodes d'interprétations, si elles appartiennent au constitutionnalisme classique et peuvent sembler anachroniques et en contradiction avec l'interprétation interculturelle prônée par le juge constitutionnel bolivien, ne constituent ici que les premières étapes d'interprétation constitutionnelle. Surtout, ce ne sont plus les méthodes d'interprétation suprêmes utilisées par le juge constitutionnel. Pour María Attard Bellido, au contraire, ces deux méthodes classiques liées à l'herméneutique sont subordonnées à la méthode de la pondération des principes, qui est utilisée en dernier recours par le juge, et qui détermine in fine l'interprétation constitutionnelle ${ }^{32}$.

Alice Mauras rappelle que les méthodes interprétatives contenues à l'article 196.II de la Constitution de 2009 se sont vues élargies par la jurisprudence constitutionnelle, notamment avec la décision $\mathrm{n}^{\circ}$ 0850/ 2013 :

«[...] Le constituant a établi deux méthodes expresses d'interprétation, mais n'a pas interdit l'utilisation d'autres méthodes d'interprétation [...] il n'est pas nécessaire de s'orienter vers une formule préconisant le choix isolé d'une méthode. L'exercice herméneutique dans la pratique implique une tâche argumentative beaucoup plus éclectique dans laquelle existent un dialogue et une interaction des différentes méthodes $[\ldots]^{33}$. »

Dès 2013, le TCP opère une distinction qualitative de la Constitution, entre une "partie dogmatique », et une "partie organique ». La partie dogmatique serait d'application directe dans la législation nationale, tandis que la partie organique aurait une applicabilité indirecte et conditionnée au nécessaire vote d'une «loi normative »:

«Dans le contexte signalé auparavant, il doit être précisé que dans tout État constitutionnel de droit, qui est une caractéristique de l'État plurinational de Bolivie, la partie dogmatique de la Constitution se caractérise par

Plurinacional no debería tomar una decisión que afecte a los pueblos indígenas sin consulta previa »; Boris Wilson Arias López, «Bases de la interpretación intercultural en un Estado plural como el boliviano », Anuario Iberoamericano de Justicia Constitucional, $\mathrm{n}^{\circ}$ 18, 2014, p. 57.

32. María Elena Attard Bellido, Sistematización de Jurisprudencia y esquemas jurisprudenciales de Pueblos Indígenas en el marco del Sistema Plural de Control de Constitucionalidad, op. cit., p. 48.

33. "Para llegar a una labor hermenéutica coincidente con la esencia y espíritu de la Constitución, no resulta una fórmula únicamente adecuada la elección aislada de un método de interpretación constitucional, el ejercicio hermenéutico en la práctica involucra una labor argumentativa mucho más ecléctica en la cual existe un diálogo e interacción de los distintos métodos de interpretación constitucional [...]», sentence constitutionnelle plurinationale $\mathrm{n}^{\circ}$ 0850/2013, Tribunal constitutionnel plurinational, Sucre, 17 juin 2013. Cité dans : 
son application directe, c'est-à-dire que sa matérialisation et donc le phénomène de constitutionnalisation dans l'ordre juridique ne nécessitent pas de loi de développement; au contraire, à la lumière du principe de légalité qui constitue un des piliers pour l'exercice de la fonction publique et au principe de sécurité et de continuité juridiques comme axes essentiels de l'État constitutionnel de droit, la partie organique de la Constitution, pour son application, nécessite des lois organiques de développement, lesquelles doivent émaner de l'ALP $[\ldots]^{34}$. »

Le TCP poursuit ce raisonnement dans sa décision du 28 novembre 2017 , et fonde une distinction entre les normes « constitutionnellesprincipes » et les normes «constitutionnelles-règles ». Les premières sont définies comme :

«[...] la pluralité des valeurs, principes droits fondamentaux non seulement individuels (libérales et sociales), mais un large catalogue de droits et garanties principes et valeurs plurielles et collectives que la Constitution représente comme un pacte de postulats distincts et parfois contradictoires, mais qui doivent au final coexister ${ }^{35}$. »

Le juge utilise également la notion de «méta-norme », de «morale objectivée-positivée ». On peut ainsi s’interroger sur la jurisprudence du juge constitutionnel bolivien à partir de la notion schmittienne de supraconstitutionnalité. En effet, le TCP, en privilégiant certaines normes constitutionnelles vis-à-vis d'autres, réactualise d'une certaine manière la notion de supraconstitutionnalité dans le droit bolivien. Ainsi, ces normes constitutionnelles-principielles ont « une validité normative, une priorité hiérarchique et sont obligatoires au regard des normes constitutionnelles-règles et à plus forte raison avec les normes

Alice Mauras, «La consécration constitutionnelle des méthodes interprétatives en Bolivie », $7^{e}$ journée de la jeune recherche en droit constitutionnel, Paris, 23 mars 2018.

34. «En el contexto antes señalado, debe precisarse que en todo Estado Constitucional de Derecho, que es un elemento que caracteriza al Estado Plurinacional de Bolivia, la parte dogmática de la Constitución, se caracteriza por su directa aplicación, es decir, que su materialización y por ende el fenómeno de constitucionalización en el ordenamiento jurídico no necesita ley de desarrollo previa; por el contrario, a la luz del principio de legalidad, que constituye uno de los pilares para el ejercicio de la función pública y merced al principio de seguridad y certeza jurídica, como ejes esenciales del Estado Constitucional de Derecho, la parte orgánica de la Constitución, para su aplicación necesita leyes orgánicas de desarrollo, las cuales, para asegurar la garantía de 'reserva de leý, deben ser emanadas de la Asamblea Legislativa Plurinacional », Déclaration constitutionnelle plurinationale no 0003/2013, Tribunal constitutionnel plurinational, Sucre, 25 avril 2013.

35. «Las normas constitucionales-principios en la Constitución boliviana, son la pluralidad de valores, principios, derechos fundamentales no sólo individuales (liberales y sociales) sino un amplio catálogo de derechos y garantías, principios y valores plurales y colectivos que la Constitución representa como un pacto de postulados distintos y hasta veces contradictorios, pero que al final deben coexistir ", sentence constitutionnelle plurinationale $\mathrm{n}^{\circ}$ 0084/2017, Tribunal constitutionnel plurinational, Sucre, 28 novembre 2017. 
légales-réglementaires (contenues dans les lois) par le seul fait d'être présent dans la Constitution ${ }^{36}{ }$. Ces normes, contenues dans la partie dogmatique de la Constitution (avec les principes, les valeurs, les droits et les garanties) ont ainsi un « effet d'irradiation et de transversalité dans le reste des normes constitutionnelles et dans tout l'ordonnancement juridique $^{37} »$.

Le TCP en tire la conclusion que les droits politiques sont du ressort des normes constitutionnelles-principielles, tandis que la limitation des mandats des organes exécutifs se caractériserait par des normes constitutionnelles-règles, inférieures dans la hiérarchie des normes constitutionnelles. En cas de contradiction entre plusieurs normes constitutionnelles - comme c'est le cas pour le juge constitutionnel - il faut donner une " primauté interprétative absolue » à ces normes constitutionnelles-principielles sur le reste de la Constitution. Le TCP établit de ce fait une hiérarchie entre les normes constitutionnelles présentes dans le bloc de constitutionnalité bolivien, et admet qu'il existe des normes constitutionnelles « inconstitutionnelles » :

«Face à la possibilité de l'existence de normes de la Constitution "inconstitutionnelles", il faut établir si sur ce type de normes il est possibilité d'exercer un contrôle de constitutionnalité et quel organe peut l'exercer, en prenant en compte que cette attribution ne se trouve pas expressément inscrite dans le texte constitutionnel ${ }^{38}$.»

Le TCP s'octroie de cette manière une compétence ex nibilo, prévue ni par la Constitution bolivienne et encore moins par le constituant bolivien. L'argumentation développée par le juge constitutionnel bolivien se fait au prix d'une interprétation très extensive de ses pouvoirs.

36. «Las normas constitucionales-principios, establecidas en el texto constitucional tienen validez las normas constitucionales-principios, establecidas en el texto constitucional », sentence constitutionnelle plurinationale $n^{\circ} 0084 / 2017$, Tribunal constitutionnel plurinational, Sucre, 28 novembre 2017.

37. «Las normas constitucionales-principios, tienen un efecto de irradiación y transversalidad en el resto de las normas constitucionales y todo el ordenamiento jurídico », sentence constitutionnelle plurinationale $\mathrm{n}^{\circ}$ 0084/2017, Tribunal constitutionnel plurinational, Sucre, 28 novembre 2017.

38. "Ahora bien, frente a la posibilidad de la existencia de normas de la Constitución "inconstitucionales", corresponde establecer si sobre este tipo de previsiones, es posible ejercer el control de constitucionalidad y cuál el órgano llamado a hacerlo, tomando en cuenta que esta atribución no se encuentra inscrita expresamente en el Texto Constitucional », sentence constitutionnelle plurinationale $\mathrm{n}^{\circ} 0084 / 2017$, Tribunal constitutionnel plurinational, Sucre, 28 novembre 2017. Le TCP signale qu'il a déjà procédé de la sorte, avec la décision 0003/2013 du 25 avril 2013 relatif à la Loi d'application normative, où le juge constitutionnel admet que l'ALP peut «matérialiser » certaines dispositions de la Constitution afin d'éviter la coexistence de normes constitutionnelles contradictoires. 


\section{B - SUPPRIMER DES NORMES CONSTITUTIONNELLES ? LA COMPÉTENCE EX NIHILO DU TCP}

En s'appuyant sur les doctrines allemande et espagnole qui admettraient déjà l'inconstitutionnalité de certaines dispositions constitutionnelles $^{39}$, le juge constitutionnel bolivien s'octroie donc la compétence de statuer sur la constitutionnalité des dispositions contenues dans le texte constitutionnel.

Nous pouvons nous interroger sur la manière dont le juge constitutionnel peut souverainement s'octroyer la compétence de supprimer certaines normes constitutionnelles qui seraient en contradiction avec le reste du texte constitutionnel. Le juge s'appuie sur l'article 196.I de la CPE qui définit les fonctions du TCP et en fait une interprétation très extensive :

« Le juge constitutionnel affirme en ce sens qu'il doit veiller à l'harmonie et à la cohérence des normes qui font partie de la Constitution ; par conséquent, à partir de la disposition constitutionnelle citée auparavant, il est possible d'assumer l'existence d'une "faculté étendue" pour que le Tribunal réalise un contrôle de constitutionnalité des propres normes constitutionnelles en prenant garde qu'il n'existe pas de normes dissonantes avec les valeurs suprêmes, les principes fondamentaux, les droits et les garanties qui consacrent l'ordre constitutionnel, garantissant ainsi l'harmonie et la cohérence en ces termes ${ }^{40}$. »

Ce n'est pas la première fois que le juge constitutionnel bolivien s'octroie des compétences d'interprétation qui ne figurent pas dans la Constitution. Ainsi, le TCP a reconnu à l'ALP un pouvoir constituant dérivé, en particulier au niveau de l'interprétation du texte constitutionnel $^{41}$. On pense ainsi à la Loi d'application normative votée en 2013 et réinterprétant la Disposition transitoire première relative au décompte des mandats présidentiels. En ce sens, le TCP argue de la nécessaire

39. «Los precitados antecedentes doctrinarios y jurisprudenciales alemanes fueron recogidos también por la doctrina española, la cual se refleja en trabajos como "En Torno a la Posible Inconstitucionalidad del Apartado Primero del Art. 57 de la Constitución Española de 1978", donde se concluye que la Constitución española de 1978 contiene normas inconstitucionales », sentence constitutionnelle plurinationale $n^{\circ} 0084 / 2017$, Tribunal constitutionnel plurinational, Sucre, 28 novembre 2017.

40. «[...] debe velar por la armonía y coherencia de las normas que lo conforman, por lo que a partir de la disposición constitucional antes citada, es posible asumir la existencia de una "facultad extendida", para que este Tribunal realice el control de constitucionalidad de las propias normas constitucionales, cuidando que en todo su entramado, no existan normas disonantes con los valores supremos, principios fundamentales derechos y garantías que consagra el orden constitucional, garantizando armonía y coherencia en sus términos », sentence constitutionnelle plurinationale $n^{\circ} 0084 / 2017$, Tribunal constitutionnel plurinational, Sucre, 28 novembre 2017.

41. Déclaration constitutionnelle plurinationale $n^{\circ} 0003 / 2013$, Tribunal constitutionnel plurinational, Sucre, 25 avril 2013. 
cohésion et contextualisation de la Constitution par l'organe législatif. Le pouvoir législatif se concevrait comme un "pouvoir constituant dérivé », car l'organe représentatif du peuple souverain devrait pouvoir dépasser les contradictions apparentes du texte constitutionnel. La loi devient donc un outil de développement et de dépassement des supposées contradictions du pouvoir constituant originaire. Ainsi, s'il y a des contradictions dans la Constitution, ce n'est pas au TCP de les déterminer ni de les interpréter, mais au pouvoir législatif qui est alors considéré comme un pouvoir constituant dérivé. Il y a ici un renversement de la hiérarchie des normes, avec une subordination de la Constitution à la loi.

Sylvie Salles propose le concept du conséquentialisme ${ }^{42}$ pour décrire la prise en compte par le juge constitutionnel des conséquences politicojuridiques de ses décisions. Salles constate la présence de considérations extrajuridiques dans les délibérations et les arguments du Conseil constitutionnel ${ }^{43}$, et plus généralement pour l'ensemble des juges constitutionnels. Ainsi, les arguments conséquentialistes extrajuridiques - qui peuvent être économiques, budgétaires, culturels, sociaux ou environnementaux - constituent le cœur du débat du Conseil constitutionnel, et fonde de manière implicite le raisonnement des décisions ${ }^{44}$.

En ce sens, nous pouvons appliquer l'hypothèse conséquentialiste à la jurisprudence du TCP. Tout comme le juge constitutionnel français, on ne saurait trouver dans les décisions d'arguments conséquentialistes. Par ailleurs, les délibérations n'étant pas rendues publiques au TCP, il nous faut chercher d'autres sources d'information; en l'occurrence, l'entretien avec d'anciens membres du TCP. Ligia Velásquez évoque ainsi l'existence d'une «interprétation sociale ${ }^{45}$ » dans la jurisprudence constitutionnelle bolivienne. Il faut ainsi prendre compte «la situation

42. Sylvie Salles, «La présence de l'argument conséquentialiste dans les délibérations du Conseil constitutionnel [en ligne] ",VIII Congrès français de droit constitutionnel, AFDC, Nancy, 16-18 juin 2011. Disponible sur : http://www.droitconstitutionnel.org/congresNancy/comN3/sallesT3.pdf

43. Par exemple, René Coty, hostile aux positions du rapporteur M. Gilbert-Jules sur l'extension de la saisine à l'ensemble de la loi et non plus à certaines dispositions soulevées par la saisine a priori. Le compte rendu de la séance rapporte que René Coty « insiste sur la gravité des conséquences s'il en était décidé autrement». Cité dans : Salles Sylvie, «La présence de l'argument conséquentialiste dans les délibérations du Conseil constitutionnel », op. cit.

44. Cependant, le juge constitutionnel français, reste formaliste dans ses décisions. Il faut alors chercher du côté des délibérations pour trouver la preuve conséquentialiste. En effet, il doit anticiper les conséquences de sa décision, qui doit être raisonnable au regard de ses effets. Si le juge français doit donc être conséquentialiste de manière officieuse, en coulisse, il n'en est pas de même avec le juge bolivien. Il faut toutefois avoir conscience de la difficulté d'apprécier les faits, et de ne pas caler le droit au fait. C'est donc avec précaution que le juge doit prendre en compte les éléments de fait.

45. Entretien avec Ligia Velásquez, ancienne membre du Tribunal constitutionnel plurinational, Cochabamba, $1^{\text {er }}$ février 2017. 
dans lequel se situe la décision ${ }^{46}$ ", à travers l'appréciation d'éléments politiques et sociaux. Les décisions du TCP ont des conséquences politiques et sociales, il faut donc chercher à savoir si la décision « résout un problème ou provoque le chaos, car elle peut porter préjudice à la société $^{47}$ ». Il s'agit donc, pour reprendre les mots de Velásquez, d'une «analyse intégrale». Le TCP est un instrument «juridique et politique », par conséquent il est nécessaire de faire l'analyse de l'histoire, des rapports de force, de l'influence de la société sur le comportement du juge constitutionnel, car ces différents facteurs vont considérablement peser sur la décision finale.

La thèse conséquentialiste, bien qu'imprécise dans le cas bolivien du fait de l'absence de publicité des délibérations du $\mathrm{TCP}^{48}$, permet d'expliquer un certain nombre de décisions peu convaincantes si on les considère à l'aune de l'interprétation exégétique ou systématique, mais pertinentes si l'on prend le conséquentialisme comme grille d'analyse. De ce fait, on peut considérer la décision $n^{\circ} 0084 / 2017$ du 28 novembre 2017 comme purement conséquentialiste.

Cette interprétation, bien qu'elle développe une argumentation étayée, n'en reste pas moins controversée. Cependant, si l'on considère cette décision à l'aune du conséquentialisme, la décision nous apparâ̂t logique, mais également légitime. En effet, si le juge constitutionnel avait refusé de revenir sur l'article 168 qui limite à deux le nombre de mandats présidentiels, on peut considérer que les conséquences politiques auraient été toutes autres, en changeant en particulier le scénario de l'élection présidentielle de décembre 2019.

Le TCP conclu donc à l'inconstitutionnalité des articles relatifs à la limitation de l'ensemble des mandats exécutifs :

«Le contenu des articles 156, 168, 285.II et 288 de la CPE, qui sont des normes constitutionnelles-règles relatives à la possibilité d'être réélu "pour une seule fois de manière continue" contredisent ou s'opposent à ce qui est établi par les articles 26 et 28 de la même constitution qui sont des normes constitutionnelles-principielles, car les premiers articles cités imposent une limitation ou une restriction dans l'octroi ou l'exercice des droits politiques consacrés dans les seconds articles cités [...] En conséquence, dans le cas de cette analyse, il résulte de manière évidente la présence d'une contradiction ou d'une antinomie [...] qui doit se résoudre en faveur des normes constitutionnelles-principielles en déterminant leur application prioritaire face aux normes constitutionnelles-règles ${ }^{49}$. "

46. Ibidem.

47. Ibidem

48. Certaines décisions comportent des opinions minoritaires; ces dernières restent cependant marginales.

49. «La previsión contenida en los arts. 156, 168, 285.II y 288 de la CPE, normas constitucionales-reglas, respecto a la posibilidad de ser reelecta o reelecto " $[\ldots]$ por una sola vez de manera continua", contradicen o se oponen a lo que establecen los arts. 26 y 28 de la misma Constitución, normas constitucionales-principios, pues los artículos en primer 
La décision du TCP a été contestée par la plupart des constitutionnalistes boliviens, sur le fond, mais également sur la forme ( « fraude procédurale »). Il s'avère ainsi que la décision $\mathrm{n}^{\circ} 0084 / 2017$, rendue le 28 novembre 2017 , était présente dans une décision rendue deux mois auparavant. Le TCP s'est appuyé sur la décision $n^{\circ}$ 0084/2017 dans le corps de la décision $n^{\circ}$ 0996/2017 relative à une action d'amparo constitutionnelle portée par Samuel Doria Medina, et rendue le 25 septembre 2017. Des actions en nullité ont bien été présentées au TCP afin d'annuler la décision $\mathrm{n}^{\circ}$ 0084/2017; elles ont toutes été rejetées par la commission d'admission du TCP. Ainsi, selon le constitutionnaliste Carlos Alarcón, cette décision « est la preuve irréfutable qui prouve un échec de la procédure, car elle cite une décision inexistante, la décision $n^{\circ} 084^{50} »$.

Ce qui distingue le constitutionnalisme latino-américain, et en particulier le constitutionnalisme bolivien, c'est la fragilité et la vulnérabilité des pouvoirs législatifs et judiciaires face au pouvoir exécutif et surtout face à la fonction présidentielle. Le constitutionnaliste José Antonio Rivera Santiváñez ${ }^{51}$ explique que la principale menace de ce nouveau constitutionnalisme est de générer un phénomène de « déconstitutionnalisation » caractérisé par un manque de loyauté envers la Constitution de la part des gouvernants. Ainsi, l'État ne respecte pas la volonté du constituant en ne mettant pas en œuvre les dispositions législatives et réglementaires pour rendre effectifs les droits inscrits, et en prenant des décisions en contradiction avec la Constitution. Le TCP, transgressant la Constitution de 2009, a donc décidé de passer outre la volonté du peuple exprimée lors du référendum de 2016. Le risque est grand de voir la crédibilité du juge constitutionnel et par extension de l'ordre judiciaire s'effriter.

Si la Constitution bolivienne est de ce fait inopérante, on peut craindre que le rapport de force sorte du champ des institutions pour déboucher sur des formes de contestation plus violentes.

término señalados, imponen una limitación o restricción, en el goce y ejercicio de los derechos políticos consagrados en los artículos en último término señalados [...] En consecuencia, en el caso en análisis, resulta evidente la presencia de una contradicción o antinomia [...] lo que conforme a lo establecido precedentemente y a la comprensión desarrollada en el Fundamento Jurídico III.2. de la presente Sentencia Constitucional Plurinacional, debe resolverse en favor de las normas constitucionales-principios, determinando su aplicación preferente frente a las normas constitucionales reglas »; sentence constitutionnelle plurinationale $n^{\circ}$ 0084/2017, Tribunal constitutionnel plurinational, Sucre, 28 novembre 2017.

50. «Es la prueba contundente que demuestra el fallo procesal porque cita una sentencia inexistente, la 084 »; María Carballo, «Identifican 4 vías para dejar sin efecto fallo de reelección [en ligne] », Pagina Siete, 7 février 2018. Disponible sur : http://www.paginasiete.bo/nacional/2018/2/7/identifican-vas-para-dejar-efecto-fallo-reeleccin-169153.html

51. José Antonio Rivera Santiváñez, «Avances, riesgos y desafíos del constitucionalismo latinoamericano ", Segundo Congreso Boliviano de Derecho Constitucional. Realidad y perspectivas del constitucionalismo boliviano, La Paz, Kipus, 2014, p. 141-162. 